\title{
Editorial
}

\section{RFID Technology and Applications}

\author{
Xiaodong Chen, ${ }^{1}$ Junsheng Yu, ${ }^{2}$ Yuan Yao, ${ }^{2}$ Chaowei Wang, ${ }^{2}$ and Daniel Valderas ${ }^{3}$ \\ ${ }^{1}$ School of Electronic Engineering and Computer Science, Queen Mary University of London, London E14NS, UK \\ ${ }^{2}$ School of Electronic Engineering, Beijing University of Posts and Telecommunications, Beijing 100876, China \\ ${ }^{3}$ Department of Electronics and Communications, University of Navarra, 31080 Navarra, Spain \\ Correspondence should be addressed to Xiaodong Chen; xiaodong.chen@eecs.qmul.ac.uk
}

Received 9 April 2014; Accepted 9 April 2014; Published 28 April 2014

Copyright (C) 2014 Xiaodong Chen et al. This is an open access article distributed under the Creative Commons Attribution License, which permits unrestricted use, distribution, and reproduction in any medium, provided the original work is properly cited.

RFID is emerging as one of most fundamental technologies to Internet of Things due to its attractive features such as good reading ranges, high data rates, and low cost. RFID has achieved a widespread success in various applications, ranging from asset tracking, highway toll collection, supply chain management, animal identification to surveillance systems. Moreover, there has been a wide scope of development in RFID standards, protocols, and middleware. However, a series of challenging issues should be properly addressed before a massive adaptation of RFID technology.

In this special issue, we have received 24 paper submissions and accepted 9 papers finally according to the reviewers' comment and associate editors' suggestion. These accepted papers include RFID reader antenna design, RFID tag antenna design, RFID passive transponders, tag collision algorithm, and RFID application systems.

B. Lee et al. from Kwangwoon University of Korea proposed a novel structure of an NFC loop antenna for mobile handset applications by improving the performance of an NFC loop antenna when a ferrite-polymer composite is attached between the embedded NFC loop antenna and the phone battery. The proposed loop antenna gives better performance than that of a conventional NFC loop antenna.

L. X. Zheng et al. from South China University of Technology proposed a compact RFID reader antenna for UHF near-field and far-field operations. The structure of the antenna is a novel folded-dipole loop and is formed by a concentric metal ring with a split. It can provide uniform magnetic near-field distribution and available farfield gain. Y. Yao et al. from Beijing University of Posts and Telecommunications also proposed a novel RFID reader antenna. This antenna was fabricated using indium tin oxide film; thus, it has optically transparent characteristic. This kind of antenna can be used in clothing stores.

P. Jankowski-Mihulowicz et al. from Rzeszów University of Technology proposed novel RFID passive transponders with additional energy harvester. They can recover energy from the electromagnetic field of read device and the harvested energy was utilized to supply a microprocessor acquisition block for developed LTCC pressure sensor.

C. Wang et al. from Beijing University of Posts and Telecommunications proposed an advanced dynamic framed-slotted ALOHA algorithm based on Bayesian estimation and probability response to solve the tag collision problem of RFID system. The simulation results show that the proposed algorithm has better performance.

We sincerely hope that this special issue can further help the readers to understand RFID antenna design and applications and explore the future development of the system.

Xiaodong Chen Junsheng Yu Yuan Yao Chaowei Wang Daniel Valderas 

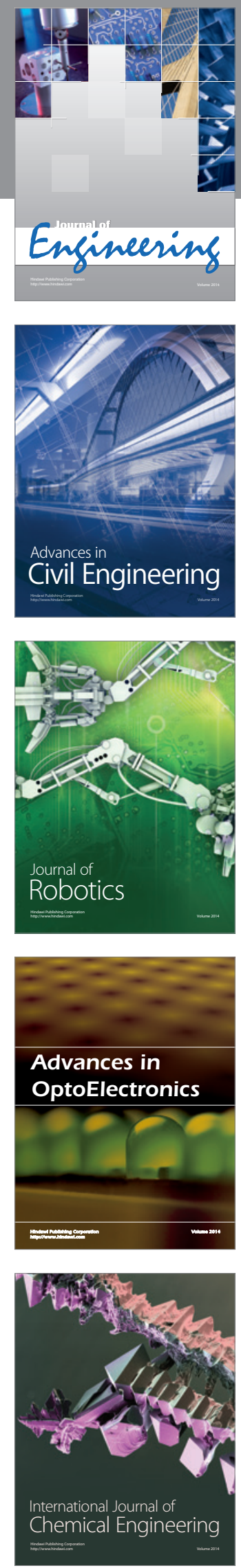

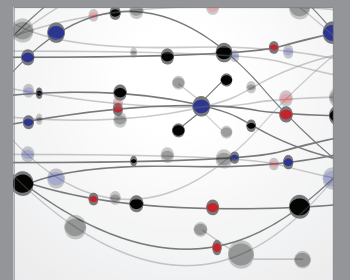

The Scientific World Journal
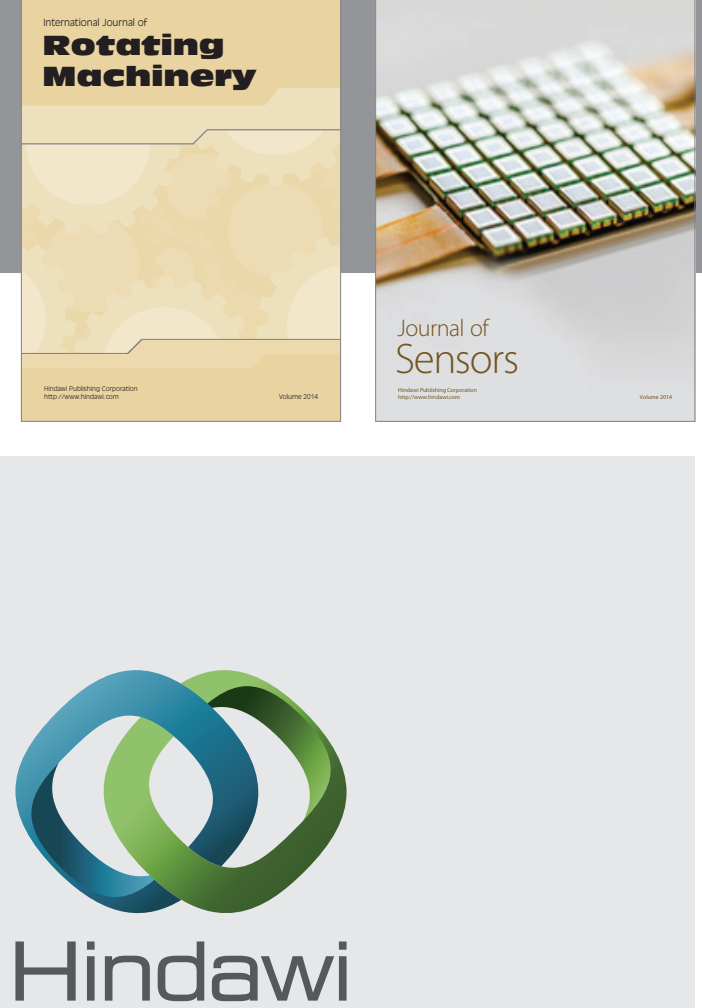

Submit your manuscripts at http://www.hindawi.com
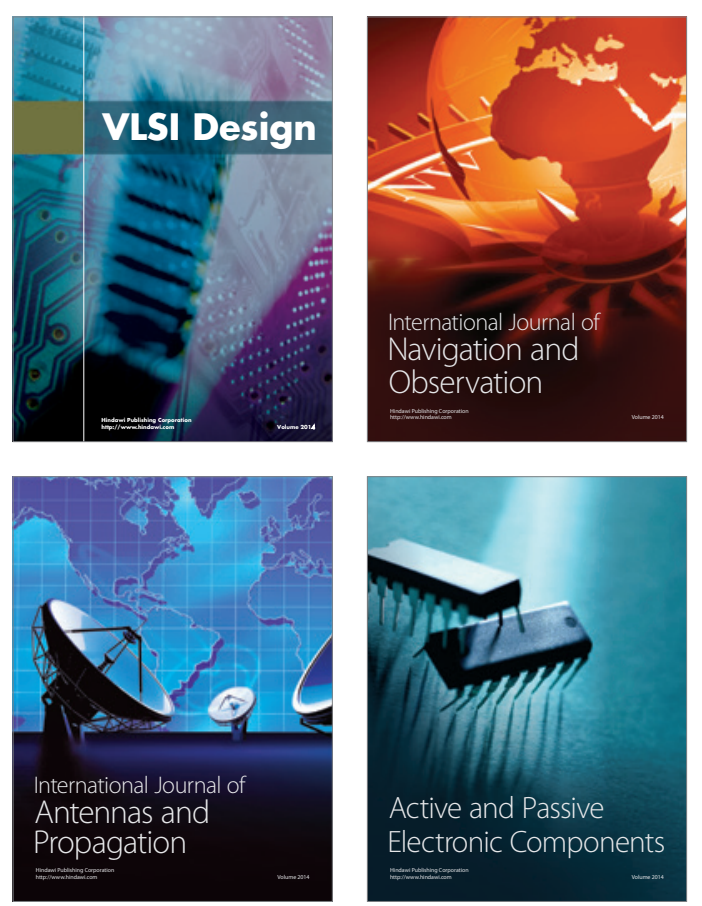
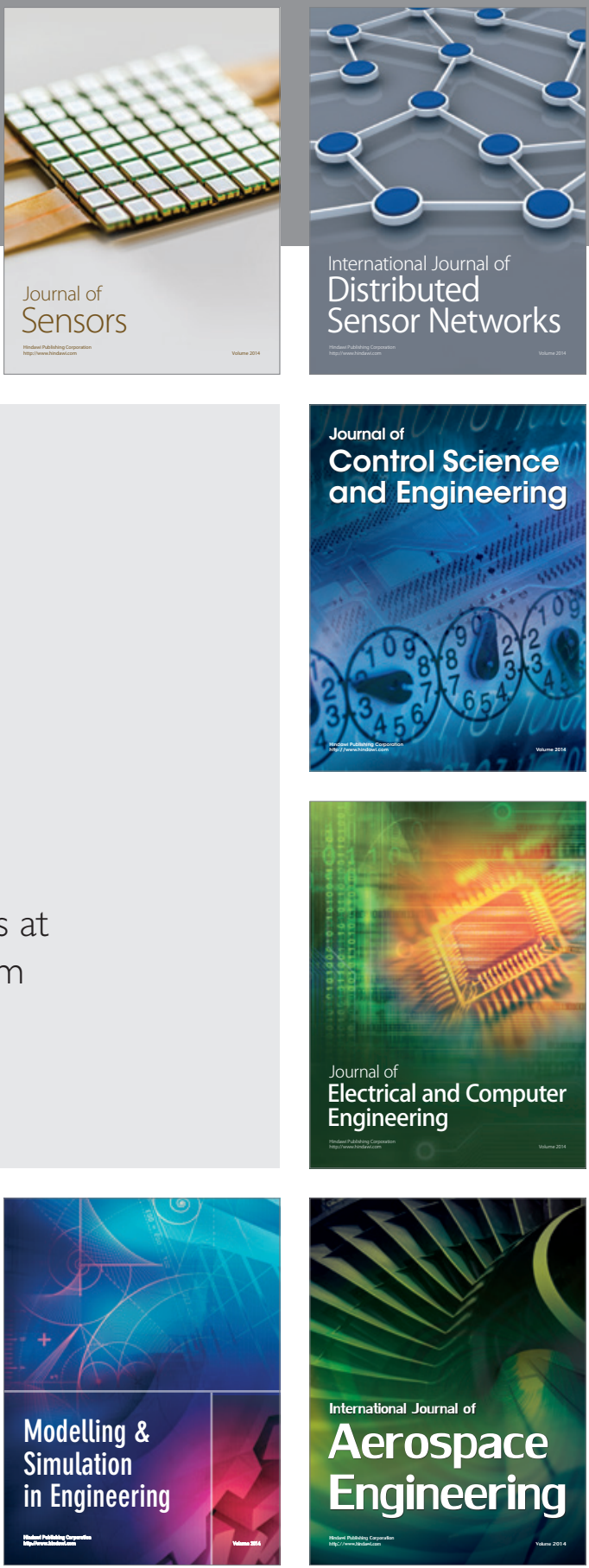

Journal of

Control Science

and Engineering
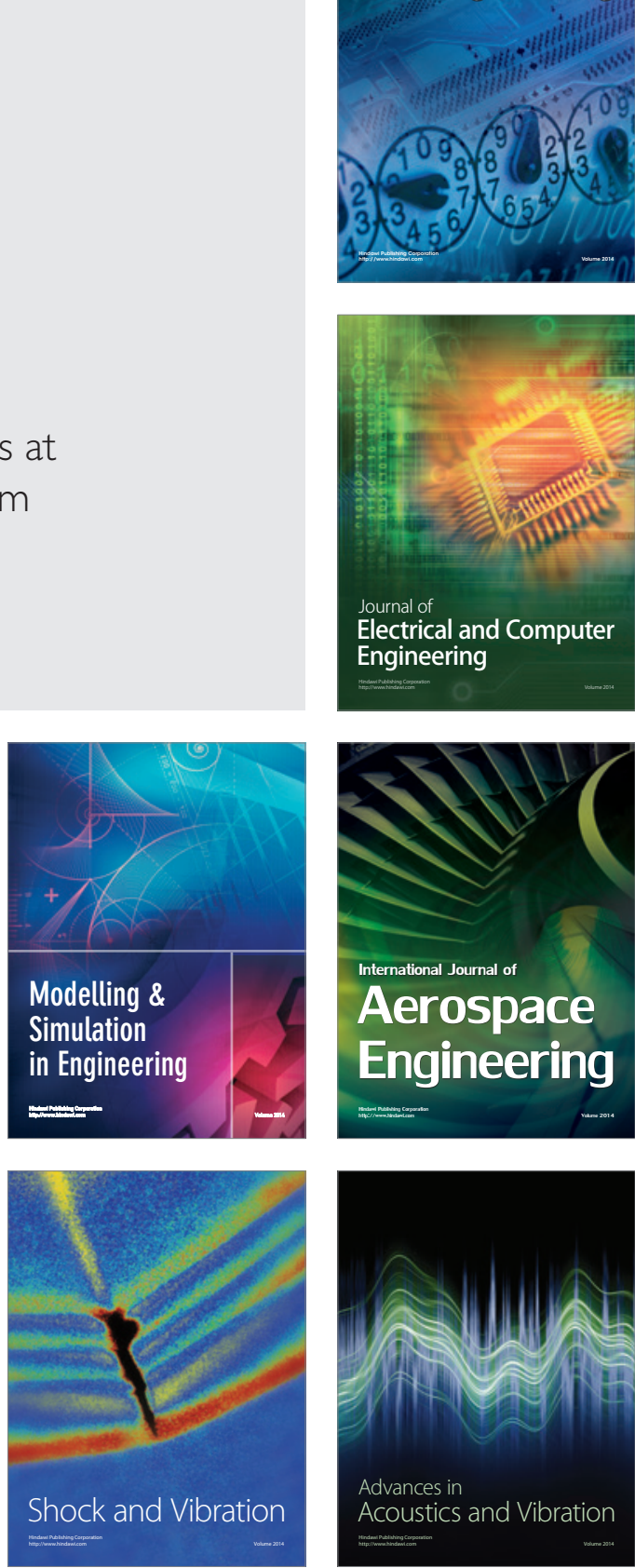